\title{
Validez factorial de la adaptación al español del Maslach Burnout Inventory-General Survey
}

Pedro R Gil-Monte, PhD. ${ }^{(1)}$

\section{Gil-Monte PR. Validez factorial de la adaptación al español del Maslach Burnout Inventory General Survey. Salud Publica Mex 2002;44:33-40.} El texto completo en inglés de este artículo está disponible en: http://www.insp.mx/salud/index.html

\section{Resumen}

Objetivo. Explo rar la estructura facto rial de una adaptación al castellano del "Maslach Burnout Inventory-General Survey" (MBI-GS). Material y métodos La muestra la integraron 149 po licías municipales deTenerife, España, a quienes se les aplicó el cuestionario en 1999. Se realizó un análisis factorial mediante Componentes Principales y rotación Varimax. Resultados Cuatro factores tuvieron eigenvalues mayores que 1. En el Factor I se agruparon los items de Eficacia Profesional, en el Factor II tres items de Cinismo, en el Factor III los items de A gotamiento, y en el Factor IV dos items de Cinismo. Posterio rmente se realizó otro análisis ajustando a tres la extracción de factores. La solución factorial reprodujo la distribución de los items del manual. Conclusiones A la luz de los resultados obtenidos el cuestionario se puede considerar válido y confiable en la versión adaptada al castellano. El texto completo en inglés de este artículo está disponible en: http://www.insp.mx/salud/ index.html

Palabras clave: cuestionario; validez; salud o cupacional; análisis factorial; España

\author{
Gil-Monte PR. \\ The Factorial Validity of the Maslach Burnout \\ Inventory-General Survey \\ (MBI-GS) Spanish Version. \\ Salud Publica Mex 2002;44:33-40. \\ The English version of this paper \\ is available at: http://www.insp.mx/salud/index.html
}

\section{Abstract}

Objective. To explore the factorial structure of a Spanish version of the "Maslach Burnout Inventory-G eneral Survey" (MBI-GS). Material and Methods. A cross-sectional study was conducted in 1999 among 149 municipal policemen in Tenerife, Spain. A questionnaire was applied to collect data on Professional Efficacy, Cynicism, and Burnout. D ata were analyzed using factorial analysis and principal components with Varimax rotation. Results. Four factors had eigenvalues greater than 1; Factor 1 go uped Professional Efficacy items, Factor II grouped three Cynicism items. Further analysis was conducted to limit extraction to three factors. The factorial solution replicated the distribution of items in the questionnaire manual. Conclusions. Study results whow that the Spanish version of the questionnaire is valid and reliable.The English version of this paper is available at:http:/ /www.insp.mx/salud/index.html

Key words:questionnaires; validity; occupational health; factor analysis, statistical; Spain

Fecha de recibido: 10 de enero de 2001 - Fecha de aprobado: 29 de junio de 2001 Solicitud de sobretiros: Pedro R. Gil Monte, Area de Psicología Social, Facultad de Psicología. Universidad de Valencia. Avenida Blasco Ibáñez, 2, 46010 Valencia, España. correo electrónico:Pedro.Gil-Monte@uv.es 
A ctualmente, pocos cuestionan que el estrés deri$A$ vado de la realización del trabajo es una de las principales causas de enfermedad laboral y de absentismo, y que da origen a multitud de accidentes. La prevención de los riesgos psicosociales en el trabajo, susceptibles de generar estrés, y de los accidentes laborales, ha cobrado un gran protagonismo social en estos últimos años en España. La aprobación de la actual Ley de Prevención de Riesgos Laborales (Boletín Oficial del Estado 10-11-1995), al reconocer la organización y ordenación del trabajo como condiciones de trabajo susceptibles de producir riesgos laborales, recoge el interés de académicos y profesionales por incorporar los riesgos de origen psicosocial entre las causas de accidentes y bajas laborales. Junto a esta ley, el Reglamento de los Servicios de Prevención (Boletín Oficial del Estado 31-1-1997) al fomentar la creación de un gran número de cursos "Máster en Prevención de Riesgos Laborales", en los que se incorpora una especialidad de "Ergonomía y Psicosociología Aplicada" y con frecuencia un módulo o unidad sobre estrés laboral, está concienciando a empresarios y prevencionistas de la importancia que tiene evaluar y prevenir el proceso de estrés para disminuir los accidentes laborales y el ausentismo en el trabajo, y para mejorar la salud y la calidad de vida laboral de todos los trabajadores.

El síndrome de quemarse por el trabajo, entendido como una respuesta al estrés laboral crónico, ha cobrado protagonismo en este contexto. Los cambios ocurridos en el entorno socioeconómico y en el mercado laboral han contribuido significativamente al desarrollo y a la extensión del síndrome de quemarse por el trabajo en la actualidad. Entre estas transformaciones se encuentran los cambios en la estructura de los sectores económicos, que han originado un fuerte crecimiento del sector servicios, y la aparición de nuevas formas de trabajo caracterizadas por la necesidad de trabajar más tiempo en contacto con las personas hacia las que se dirige el trabajo (v.g., clientes y usuarios de la organización). Asimismo, el auge de las organizaciones y actividades de voluntariado, en las que está implicada gran parte de la población, ha hecho que el fenómeno se difunda.

El síndrome de quemarse por el trabajo se ha conceptualizado como un proceso que ocurre entre los profesionales que trabajan hacia personas, o cuyo objeto de trabajo son personas (v.g., trabajadores pertenecientes a los sectores de la sanidad, educación, hostelería, justicia, seguridad, servicios sociales, etcétera). A raíz de los trabajos de C. Maslach y S. Jakcson, el fruto fue el Maslach Burnout Inventory en su versión para los profesionales de "servicios humanos"
(MB1-HSS); ${ }^{*}$ el fenómeno fue entonces definido como un síndrome caracterizado por la aparición de baja realización personal en el trabajo (tendencia a evaluarse negativamente, de manera especial con relación a la habilidad para realizar el trabajo y para relacionarse profesionalmente con las personas a las que atiende), alto agotamiento emocional (no poder dar más de sí mismo en el ámbito emocional y afectivo) y alta despersonalización (desarrollo de sentimientos y actitudes de cinismo y, en general, de carácter negativo hacia las personas destinatarias del trabajo). ${ }^{3}$

No obstante, el síndrome de quemarse por el trabajo no está necesariamente restringido a los profesionales de los servicios humanos. Maslach y Schaufeli ${ }^{4}$ señalan que aunque este síndrome aparece como un proceso específico de estos ámbitos profesionales, el fenómeno ha sido descrito en otro tipo de profesionales, como directivos y mandos intermedios de cualquier tipo de organización, entrenadores y deportistas, e incluso ha sido estudiado fuera del ámbito laboral (v.g., en amas de casa). Sin embargo, evaluar el síndrome de quemarse por el trabajo en estos trabajadores mediante el MBI-HSS supone un gran problema. Su evaluación, según el MBI, fue concebida para evaluar únicamente a profesionales de servicios y sus items hacen alusión a las personas destinatarias del trabajo (v.g., pacientes o alumnos), por ello es prácticamente imposible de aplicar en las actividades en las que el trabajador no tiene un contacto directo con el cliente o usuario de la organización.

Para resolver este inconveniente Schaufeli, Leiter, Maslach, y Jackson ${ }^{5}$ elaboraron una escala que permite evaluar el síndrome en cualquier tipo de trabajadores. A diferencia del MBI-HSS los items del Maslach Burnout Inventory-General Survey (MBI-GS) no se refieren explícitamente a las personas destinatarias del trabajo, tienen un carácter más genérico no exclusivo para profesionales de servicios. ${ }^{6}$ Cuando se evalúa el síndrome de quemarse por el trabajo mediante el MBIGS estamos evaluando una crisis en la relación de una persona con su trabajo, y no necesariamente una crisis en las relaciones entre una persona y la gente con la que se relaciona en el trabajo. Se asume que la relación

\footnotetext{
* El MBI-HSS es la versión inicial del MBI, y se alude a él como MBI-HSS o MBI, indistintamente. Sin embargo, en la última edición del manual ${ }^{1}$ los autores establecen la necesidad de identificar las diferentes versiones comercializadas del MBI: a) el MBI-Human Services Survey (MBI-HSS), dirigido a los profesionales de la salud, b) el MBI-Educators Survey (MBI-ES), la versión del MBI-HSS para profesionales de la educación ${ }^{2}$, que se diferencia de la anterior en que se sustituye la palabra paciente por alumno en los items, y c) el MBI-General Survey (MBI-GS).
} 
de las personas con su trabajo se sitúa sobre un continuo que va de "compromiso con el trabajo" (engagement) a "quemarse por el trabajo" (burnout). El compromiso con el trabajo es un estado genérico en el que un trabajador se empeña en realizar su trabajo de manera excelente y experimenta altos sentimientos de efectividad. Por el contrario, quemarse por el trabajo es entendido como un estado de agotamiento en el que el trabajador se muestra cínico sobre el valor y el sentido de su trabajo, y duda completamente de su capacidad para realizarlo. ${ }^{5,6}$ Esta concepción del constructo quemarse por el trabajo abre la posibilidad de concebirlo como unidimensional frente a las recomendaciones hechas hasta ahora, en relación con el MBIHSS, de evaluarlo y mantenerlo como un constructo tridimensional, sin agregar las puntuaciones de las tres dimensiones en una puntuación única. ${ }^{7}$

El MBI-GS mantiene la estructura tridimensional del MBI-HSS, aunque sólo consta de 16 items frente a los 22 de esa escala. Esos 16 items se distribuyen en las tres dimensiones de la escala denominadas: Eficacia profesional (seis items), Agotamiento (cinco items) y Cinismo (cinco items). La subescala de Eficacia profesional es similar a la de Realización personal en el trabajo del MBI-HSS, pero recoge aspectos sociales y no sociales del trabajo, y se centra más en las expectativas de éxito del sujeto (v.g., "Creo que tengo confianza en mi eficacia para alcanzar los objetivos"). Los items que miden agotamiento son más genéricos que los de la subescala de Agotamiento emocional del MBI-HSS, pues incluyen referencias a la fatiga física y emocional sin hacer referencia a las personas que pueden ser la causa de esos sentimientos de fatiga. Por ejemplo, el item del MBI-HSS "Trabajar con pacientes todos los días es una tensión para mí" ha sido sustituido por el item "El trabajo diario es realmente una tensión para mí". La subescala de Cinismo quizá es la que presenta más diferencias de las tres con su homóloga del MBIHSS. Los items que miden cinismo, a diferencia de los que miden despersonalización, reflejan indiferencia o actitudes de distanciamiento hacia el trabajo, y no se centran en las personas hacia las que se dirige éste (v.g., "Pienso que he perdido el entusiasmo por mi profesión" o "Sólo deseo hacer mi trabajo y que no me molesten"). Pero, al igual que en el caso de la despersonalización, se considera que evalúan el intento de los individuos de distanciarse del trabajo como estrategia de afrontamiento frente a las exigencias agotadoras de éste, y las actitudes de cinismo pueden ser una estrategia de afrontamiento disfuncional, pues reducen la energía del individuo para hacer un buen trabajo y para desarrollar soluciones creativas a los problemas laborales.
Aunque el MBI-HSS y el MBI-GS tienen muchos aspectos en común, la diferencia de fondo más relevante entre ambos instrumentos de medida es que el MBI-GS no se centra en la relación laboral de servicio que se establece entre un profesional y los usuario o clientes, sino en la realización del trabajo en general.

La estructura factorial del MBI-GS ha sido contrastada en algunos estudios mediante análisis factorial confirmatorio. Una primera versión propuesta para la escala contenía 24 items, ${ }^{8}$ pero posteriores estudios confirmatorios recomendaron reducir la escala a 16 items. Es el caso del estudio de Leiter y Schaufeli, ${ }^{6}$ que con diferentes muestras de profesionales de la salud obtuvieron un ajuste adecuado para el modelo de tres factores referido arriba, con valores superiores a 0.90 en diferentes índices Goodness Fit Index (GFI), Delta2 y Relative Noncentraly Index (RNI). El modelo propuesto incluía relaciones entre los tres factores con paths de Agotamiento a Cinismo, y de Cinismo a Eficacia profesional, y presentó un incremento significativo en $\chi^{2}$ respecto al modelo hipotetizado con los tres factores no correlacionados. Esta estructura de tres factores también ha sido confirmada con las versiones holandesa, ${ }^{9}$ finlandesa y sueca, ${ }^{10}$ obteniéndose resultados similares a los de la versión inglesa, aunque en el estudio de Schutte y colaboradore ${ }^{10}$ el item 13 ("Sólo deseo hacer mi trabajo y que no me molesten") fue eliminado del modelo factorial debido a que disminuía considerablemente la consistencia interna de la subescala Cinismo.

En la actualidad no existe en España una versión comercial del MBI-GS, ni estudios publicados en los que se analice la estructura factorial de esta escala. La experiencia habida con el MBI-HSS hace pensar que esta nueva versión del MBI va a tener difusión y utilización similar a la de las versiones anteriores del instrumento, pues muchos profesionales y organizaciones han visto limitado su deseo de evaluar el síndrome de quemarse por el trabajo debido a que no disponían de un instrumento adecuado para ello; de aquí la importancia de proceder a la adaptación y validación de esta versión del MBI al castellano. Dadas la ausencia de estudios psicométricos previos sobre este instrumento en España y la existencia de resultados dispares obtenidos con algunas de sus adaptaciones a otras lenguas, ${ }^{10}$ y a que metodológicamente se recomienda progresar desde etapas exploratorias a otras confirmatorias en la validación factorial de escalas de medida, consideramos necesario llevar a cabo un estudio exploratorio de la validez factorial del MBI-GS como paso previo a futuros estudios en los que, mediante análisis factorial confirmatorio, se contraste su estructura factorial. Por ello, el objetivo del presente 
estudio es explorar la estructura factorial del MBI-GS para la adaptación de los items realizada por nuestro equipo de investigación sin asumir, a priori, ningún modelo factorial.

\section{Material y métodos}

La muestra del estudio está compuesta por 148 policías municipales de Tenerife, España, a quienes se les aplicó el cuestionario en 1999. Por sexos, 127 son varones $(85.8 \%)$ y 17 mujeres (11.5\%). En cuatro cuestionarios no se reflejó el sexo. La media de edad de la muestra fue $\bar{X}=34.69$ años (desviación estándar $-\mathrm{DE}-=8.59$, max. $=64$, min. $=20$ ). La media de antigüedad en la profesión fue de 9.8 años, en la organización, de 9.52 años, y en el puesto, de 8.25 años. Con relación a las variables estado civil y número de hijos, 126 sujetos contestaron que tenían pareja estable (85.1\%) y 18 que no tenían pareja estable (12.2\%), 91 tenían algún hijo $(61.5 \%)$ y 53 no tenían hijos (35.8\%). Según el tipo de contrato, 120 respondieron que tenían contrato fijo $(81.1 \%)$, siete contrato eventual (4.7\%), y 19 estaban en periodo de prácticas $(12.8 \%)$. Respecto al rango jerárquico, 118 tenían la categoría de policía sin rango (79.7\%), 11 tenían la categoría de cabo $(7.4 \%)$, dos la de sargento $(1.4 \%)$, y en 17 cuestionarios no se contestó este item (11.5\%). Atendiendo a la variable nivel de estudios, 19 respondieron que tenían estudios universitarios (licenciatura o diplomatura) (12.9\%) y 123 que no los tenían $(83.1 \%)$.

Los datos fueron recogidos mediante la adaptación al castellano del "Maslach Burnout InventoryGeneral Survey" (MBI-GS) ${ }^{5}$ elaborada por nuestro equipo de investigación. El cuestionario consta de 16 items que, según señala el manual, se distribuyen en tres subescalas denominadas Eficacia profesional (6 items), Agotamiento (5 items) y Cinismo (5 items). Los sujetos valoran cada item del cuestionario con una escala tipo Likert en la que indican la frecuencia con la que han experimentado la situación descrita en el item. Esta escala de frecuencia tiene siete grados que van de 0 ("Nunca") a 6 ("Todos los días"). Bajas puntuaciones en eficacia profesional y altas puntuaciones en agotamiento y en cinismo suponen percibirse "más quemado por el trabajo". Los valores de fiabilidad de las escalas según alfa de Cronbach en nuestro estudio fueron 0.85 para Eficacia profesional, 0.83 para Agotamiento y 0.74 para Cinismo.

Los datos fueron recogidos por los autores y colaboradores seleccionando a los sujetos de manera no aleatoria. Se distribuyeron un total de 300 cuestionarios y se recogieron 149, con lo que el porcentaje de respuesta alcanzado fue $46.67 \%$.
El análisis de los datos se realizó mediante el paquete estadístico SPSS 6.1.2 para Windows. La estructura factorial del cuestionario se evaluó mediante la opción análisis factorial. Esta técnica estadística permite resumir la información contenida en un conjunto de variables (items) en un número reducido de factores. Los factores representan a las variables originales, con una pérdida mínima de información. Se empleó el método de Componentes Principales con rotación Varimax para la extracción de los factores, y se retuvieron aquellos factores con eigenvalue mayor que 1.00.11,12 Para asignar los items a los factores se consideraron las cargas factoriales iguales o mayores que 0.50.

\section{Resultados}

Antes de realizar el análisis factorial se consideraron algunos criterios para valorar la viabilidad del mismo: la matriz de correlaciones presentó un gran número de correlaciones $(51.7 \%$ ) con un valor superior a 0.30 , el determinante de la matriz de correlaciones alcanzó un valor de 0.00039, el test Kaiser Meyer Olkin (KMO) de adecuación de la muestra fue de 0.83 , y los valores del Measures of Sampling Adequacy (MSA) se encontraron en mayor medida $(81.25 \%)$ por encima de 0.80 . Estos valores indican que es pertinente realizar un análisis factorial de la matriz de correlaciones.

Con base en el manual del MBI que asume la independencia de los factores, se empleó para factorizar el método de Componentes Principales con rotación Varimax. Como se aprecia en el cuadro I se obtuvieron cuatro factores con eigenvalue mayor que 1 , que explicaron $65.4 \%$ de la varianza total. Tomando como criterio asignar un item al factor en el que presentara una carga factorial mayor de 0.50 , en el Factor I $(37.40 \%$ de la varianza) se agruparon los items 5, 7, 10, 11, 12, y 16, que estiman eficacia profesional. En el Factor II (13.60\% de la varianza) se agruparon los items 8,9 , y 15, diseñados para medir cinismo, junto con el item 7 (con carga negativa) que en el manual estima eficacia profesional. Los items de la subescala que mide agotamiento (items 1, 2, 3, 4, y 6) se agruparon en el Factor III $(8.10 \%$ de la varianza). Por último, el Factor IV (6.30\% de la varianza) quedó definido por los items 13 y 14, diseñados para medir cinismo.

En un segundo paso, se realizó un análisis factorial exploratorio ajustando a tres el número de factores a extraer para contrastar con qué grado de ajuste nuestros datos reproducían la distribución original de los items, ofrecida en la solución trifactorial del manual. El procedimiento seguido fue también el de Componentes Principales con rotación Varimax. Este resultado aparece reflejado en el cuadro II. Los tres factores 


\section{Cuadro I \\ Matriz de Cargas factoriales para la solución DE FACTORES CON EIGEN VALUE SUPERIOR A 1 UTILIZANDO Componentes Principales y rotación Varimax. Tenerife, España, 1999}

Item

Factor I Factor II Factor III Factor IV

\begin{tabular}{|c|c|c|c|c|}
\hline 1. A gotado emocionalmente & -0.18 & 0.03 & 0.81 & 0.11 \\
\hline 2. A gotado & 0.04 & 0.07 & 0.85 & -0.04 \\
\hline 3. Cansado & -0.19 & 0.18 & 0.72 & 0.19 \\
\hline 4.Tensión & -0.02 & 0.45 & 0.59 & 0.07 \\
\hline 5. Eficacia & 0.69 & -0.24 & 0.11 & 0.11 \\
\hline 6. Q uemado & -0.22 & 0.48 & 0.59 & 0.07 \\
\hline 7. Contribución & 0.52 & -0.67 & 0.03 & 0.10 \\
\hline 8. Pérdida de interés & -0.28 & 0.75 & 0.19 & 0.14 \\
\hline 9. Pérdida de entusiasmo & -0.25 & 0.83 & 0.20 & 0.09 \\
\hline 10. Bueno en el trabajo & 0.78 & -0.07 & -0.16 & 0.03 \\
\hline 11. Estimulado & 0.73 & -0.11 & -0.21 & -0.25 \\
\hline 12. Logro & 0.75 & -0.25 & -0.18 & -0.11 \\
\hline 13. N o me molesten & 0.04 & 0.03 & 0.07 & 0.88 \\
\hline 14. Cínico & -0.10 & 0.39 & 0.16 & 0.58 \\
\hline 15. Dudas & -0.16 & 0.65 & 0.19 & 0.22 \\
\hline 16. Confianza & 0.68 & -0.38 & -0.11 & 0.02 \\
\hline Eigenvalue & 5.98 & 2.18 & 1.29 & 1.01 \\
\hline \multicolumn{5}{|l|}{ Porcentaje } \\
\hline $\begin{array}{l}(n=148) \\
\text { N ota: valores } \geq 0.50 \text { en neg }\end{array}$ & & & & \\
\hline
\end{tabular}

explicaron de manera conjunta $59.1 \%$ de la varianza total. El Factor I quedó integrado por los items de la subescala Eficacia profesional, el Factor II lo formaron los items de la subescala Agotamiento, y el Factor III lo configuraron los items de la subescala Cinismo. Como se aprecia en el cuadro II, en esta solución factorial todos los items presentaron cargas factoriales superiores a 0.55 en el que es su factor según el manual, y ninguno presentó cargas factoriales por encima de este punto en otro factor. Comrey ${ }^{13}$ ha sugerido que a partir de 0.55 las cargas factoriales pueden ser consideradas buenas, y explicarían en torno al $30 \%$ de la varianza.

En el cuadro III se recogen la media, la desviación típica y el valor alfa de Cronbach para las subescalas del MBI-GS. La media para la subescala Eficacia profesional fue 4.79 ( $\mathrm{DE}=1.15)$, para Agotamiento 2.35 (DE=1.30) y para Cinismo 1.56 (DE=1.22). Al comparar los valores de la media obtenidos en este estudio con los de otros estudios (cuadro III), observamos que la

\section{Cuadro II \\ Matriz de CARGas factoriales PARA la eStruCtura DE TRES FACTORES UTILIZANDO CoMponentes Principales y rotación Varimax. Tenerife, España, 1999}

Número de item por escalas según manual Factor I Factor II Factor III

\begin{tabular}{rrrrr} 
Eficacia profesional & 5 & 0.74 & 0.09 & -0.03 \\
\hline & 7 & 0.71 & 0.02 & -0.40 \\
\hline & 10 & 0.75 & -0.19 & 0.06 \\
\hline 11 & 0.66 & -0.24 & -0.14 \\
\hline 12 & 0.75 & -0.21 & -0.17 \\
\hline 16 & 0.75 & -0.13 & -0.19
\end{tabular}

\begin{tabular}{llrrr} 
Agotamiento & 1 & -0.13 & 0.82 & 0.08 \\
& 2 & 0.04 & 0.84 & 0.05 \\
\hline & 3 & -0.17 & 0.73 & 0.25 \\
\hline 4 & -0.13 & 0.58 & 0.41 \\
\hline 6 & -0.32 & 0.58 & 0.40
\end{tabular}

\begin{tabular}{rrrrr} 
Cinismo & 8 & -0.45 & 0.19 & 0.65 \\
\hline 9 & -0.46 & 0.19 & 0.69 \\
& 13 & 0.21 & 0.09 & 0.55 \\
\hline 14 & -0.09 & 0.17 & 0.64 \\
\hline 15 & -0.30 & 0.19 & 0.63
\end{tabular}

$(n=148)$

$N$ ota: valores $\geq 0.50$ en negrita

media en Eficacia profesional resultó significativamente más alta que las medias obtenidas para las muestras de profesionales de la salud del estudio de Leiter y Schaufeli, ${ }^{6}$ y las obtenidas para la muestra de ingenieros y de staff de universidad del estudio de Taris y colaboradores. ${ }^{9}$ No se obtuvieron diferencias significativas en relación con el valor de la media obtenida en los estudios de Schaufeli y colaboradores ${ }^{5}$ con militares, y Schutte y colaboradores ${ }^{10}$ con trabajadores de la madera. En relación con la media obtenida para la subescala de Agotamiento, resultó significativamente menor a la de los estudios de Leiter y Schaufeli, ${ }^{6}$ y significativamente mayor a la obtenida en los estudios de Schaufeli y colaboradores. ${ }^{5}$ Schutte y colaboradores, ${ }^{10}$ y Taris y colaboradores. ${ }^{9}$ Por último, con relación a la media obtenida para la subescala de Cinismo, el valor obtenido en este estudio resultó significativamente menor que los del estudio de Leiter y Schaufeli, ${ }^{6}$ y similar a los obtenidos en el resto de los estudios.

En el cuadro III se recogen también los valores alfa de Cronbach que resultaron superiores a 0.70 para to- 


\section{Cuadro III \\ Estadísticos descriptivos y valores de confiabilidad alfa de Cronbach para las escalas del MBi-GS. TenerifE, España, 1999}

\begin{tabular}{|c|c|c|c|c|c|c|c|c|c|c|c|c|}
\hline \multirow[b]{2}{*}{ Muestras } & \multicolumn{4}{|c|}{ Eficacia profesional } & \multicolumn{4}{|c|}{ Agotamiento } & \multicolumn{4}{|c|}{ Cinismo } \\
\hline & $\bar{X}$ & $\mathrm{DE}$ & $\alpha$ & Valor t (gl) & $\bar{x}$ & $\mathrm{DE}$ & $\alpha$ & Valor t (gl) & $\overline{\bar{x}}$ & $\mathrm{DE}$ & $\alpha$ & Valor t (gl) \\
\hline \multicolumn{13}{|l|}{ Este estudio } \\
\hline Policía local ( $n=148)$ & 4.79 & 1.15 & 0.85 & - & 2.35 & 1.30 & 0.83 & - & 1.56 & 1.22 & 0.74 & - \\
\hline \multicolumn{13}{|l|}{ Leiter y Schaufeli (1996) } \\
\hline Enfermería $(n=1,257)$ & 4.41 & 0.99 & 0.75 & $4.34 *(1403)$ & 2.98 & 1.38 & 0.80 & $-5.28 *(1403)$ & 1.80 & 1.24 & 0.80 & $-2.23^{\ddagger}(1403)$ \\
\hline Personal hospital $(n=3,312)$ & 4.53 & 0.99 & 0.73 & $3.10^{5}(3469)$ & 2.77 & 1.45 & 0.89 & $-2.36^{\S}(3469)$ & 1.75 & 1.26 & 0.77 & $-2.27^{\ddagger}(3469)$ \\
\hline \multicolumn{13}{|l|}{ Schaufeli y colaboradores (1996) } \\
\hline Militares ( $n=147)$ & 4.60 & 0.93 & - & $1.56(293)$ & 2.05 & 1.23 & - & $2.04^{\ddagger}(293)$ & 1.63 & 1.35 & - & $0.47(293)$ \\
\hline \multicolumn{13}{|l|}{ Schutte y colaboradores (2000) } \\
\hline Sector madera $(n=9,055)$ & 4.66 & 1.69 & 0.83 & $0.93(9201)$ & 1.48 & 1.41 & 0.86 & $7.45 *(9201)$ & 1.48 & 1.69 & 0.75 & $0.57(9201)$ \\
\hline \multicolumn{13}{|l|}{ Taris y colaboradores (1999) } \\
\hline Ingenieros $(n=179)$ & 3.54 & 0.47 & 0.69 & $13.27 *(325)$ & 1.89 & 1.00 & 0.87 & $3.61 *(325)$ & 1.74 & 1.00 & 0.74 & $-1.47(325)$ \\
\hline Staff universidad $(n=284)$ & 3.25 & 0.82 & 0.73 & $16.06^{*}(430)$ & 1.46 & 0.87 & 0.86 & $8.46 *(430$ & 1.55 & 0.96 & 0.72 & $0.09(430)$ \\
\hline $\begin{array}{l}* p<0.001 \\
\ddagger p<0.05 \\
\$ p<0.01\end{array}$ & & & & & & & & & & & & \\
\hline
\end{tabular}

das las subescalas, lo que indica que la consistencia interna de las dimensiones del instrumento es buena. ${ }^{14}$ No obstante, hay que señalar que el item 13 ("Sólo deseo hacer mi trabajo y que no me molesten") repercutía negativamente en el valor alfa de la subescala Cinismo. Mientras que la eliminación del resto de los items disminuía el valor del alfa en esta subescala por debajo de 0.70 , la eliminación del item 13 incrementaba su valor a 0.79 . El resto de items también presentó una contribución positiva al alfa de su respectiva subescala, y la eliminación de cualquier item afectaba negativamente a la consistencia interna en todos los casos.

En el cuadro IV se presentan las correlaciones entre las subescalas del MBI-GS. Se observa que la correlación $r$ de Pearson más intensa se estableció entre Agotamiento y Cinismo $(r=0.52, p<0.001)$, seguida de la correlación entre Eficacia profesional y Cinismo $(r=-$ $0.49, p<0.001)$, y la correlación menos intensa fue para la asociación entre las subescalas Eficacia profesional y Agotamiento $(r=-0.37, p<0.001)$. En el cuadro IV se aprecia que la tendencia de estos resultados es similar a los resultados obtenidos en otros estudios.$$
\text { Discusión }
$$

Como se desprende de los resultados del análisis factorial exploratorio inicial (cuadro I), la adaptación al castellano del MBI-GS presenta cuatro factores, aunque de manera global la estructura y composición de la solución factorial puede ser considerada similar a la ofrecida por el manual. Esto es, se ha obtenido un factor que agrupa los items que miden eficacia profesional (Factor I), un factor donde se agrupan los items que miden agotamiento (Factor III) y dos factores que miden cinismo (Factor II y Factor IV).

Según se deriva de la solución factorial exploratoria ajustada a tres factores (cuadro II), el Factor II y el Factor IV pueden ser considerados como dos componentes de una misma dimensión (Cinismo). En esta solución, las cargas factoriales indican que todos los items se asocian con un único factor reproduciendo el modelo de tres factores del manual. Los factores aparecen diferenciados de manera clara con relación a su unidimensionalidad y ortogonalidad. Por tanto, podemos concluir que los tres factores obtenidos son similares a los ofrecidos por el manual, y que la estructura factorial de la versión española es similar a la estructura factorial de la escala original. Estos resultados deben ser replicados mediante estudios factoriales confirmatorios, pues el hecho de que la dimensión Cinismo se haya desdoblado en dos factores puede suponer una debilidad de esta adaptación

Como se ha reflejado en la sección de resultados, los valores de la media en las dimensiones del MBI-GS 


\section{Cuadro IV \\ Valores de correlación r de Pearson para las escalas del MBI-GS. Tenerife, España, 1999}

\begin{tabular}{|c|c|c|c|c|c|c|c|c|c|c|c|c|}
\hline & $\begin{array}{c}\text { Este estudio } \\
\text { Policía local } \\
(n=148)\end{array}$ & $\begin{array}{c}\text { Leiter y } \\
\text { Schaufeli (1996) } \\
\text { Enfermería } \\
(n=1257)\end{array}$ & colabo & $\begin{array}{l}\text { Taris y } \\
\text { adores } \\
\text { genierc } \\
n=179\end{array}$ & $\begin{array}{l}\text { (1999) } \\
\text { S }\end{array}$ & $\begin{array}{r}\text { colabor } \\
\text { Staff }\end{array}$ & $\begin{array}{l}\text { Taris y } \\
\text { dores (1999) } \\
\text { universidad } \\
=284)\end{array}$ & colabo & $\begin{array}{l}\text { Chaufe } \\
\text { radores } \\
\text { Militare } \\
(n=147\end{array}$ & $\begin{array}{l}y \\
(1996 \\
\text { s } \\
\text { ) }\end{array}$ & $\begin{array}{r}\text { I } \\
\text { valor } \\
\text { para I } \\
\text { (S } \\
\text { colabor }\end{array}$ & $\begin{array}{l}\text { ntervalo } \\
\text { es obtenidos } \\
\text { a r de Person } \\
\text { chaufeli y } \\
\text { adores, 1996) }\end{array}$ \\
\hline & (1) (2) (3) & $\begin{array}{lll}\text { (1) } & (2) & \text { (3) }\end{array}$ & (1) & (2) & (3) & (1) & (2) & (1) & (2) & (3) & (1) & $(2) \quad(3)$ \\
\hline Eficacia profesional & 1.00 & 1.00 & 1.00 & & & 1.00 & & 1.00 & & & 1.00 & \\
\hline Agotamiento & $-0.37 * 1.00$ & $-0.24 * 1.00$ & $-0.22 *$ & 1.00 & & $-0.38^{*}$ & 1.00 & -0.09 & 1.00 & & $-0.04 /-0.34$ & 1.00 \\
\hline Cinismo & $-0.49 * 0.52 * 1.00$ & $-0.44 * 0.58 * 1.00$ & $-0.37 *$ & $0.43^{*}$ & 1.00 & $-0.42 *$ & $0.52 * 1.00$ & $-0.57^{*}$ & $0.51^{*}$ & 1.00 & $-0.38 /-0.57$ & $0.44 / 0.611 .00$ \\
\hline
\end{tabular}

varían significativamente en relación con los obtenidos en otros estudios, si bien esos valores han sido similares en algunos casos. Estos resultados aconsejan sobre la necesidad de elaborar criterios diagnósticos normativos específicos para la adaptación del cuestionario al castellano, así como para los diferentes colectivos profesionales. De lo contrario, podemos cometer serios errores diagnósticos en la identificación del síndrome de quemarse por el trabajo (ver Gil-Monte y Peiro ${ }^{15}$ para un análisis del problema).

El análisis de fiabilidad indica que las tres subescalas tienen buenos valores de consistencia interna. A pesar de que el item 13 disminuyó el valor del alfa en la subescala Cinismo, este valor permaneció por encima de 0.70 , por lo que el item no fue desechado en los análisis. Este resultado es similar al obtenido por Schutte y colaboradores. ${ }^{10}$ Según estos autores este resultado puede ser debido a la ambivalencia del item 13. Una alta puntuación en el item puede indicar una falta de compromiso con el trabajo y un deseo de aislamiento social pero, al mismo tiempo, una alta puntuación también puede indicar alto compromiso y alta motivación laboral, esto es, el individuo no desea ser interrumpido porque desea concentrarse en la tarea o está muy concentrado en ella. La naturaleza ambivalente del item estaría respaldada, al igual que en el estudio de Schutte y colaboradores ${ }^{10}$ por el hecho de que en el presente estudio también presentó la mayor desviación típica de los 16 items de la escala (2.29 en este estudio, y 2.22 en el estudio de Schutte y colaboradores. $)^{10}$ Además, Schaufeli y colaboradores ${ }^{8}$ obtuvieron que el item 13 tenía la carga factorial más baja en tres muestras independientes. Un resultado similar fue obtenido también por Leiter y Schaufeli. ${ }^{6}$ Este resultado también se da en nuestro estudio: en la solución de tres factores la carga factorial de este item fue
0.55. Por tanto, estudios posteriores deberían considerar la desviación típica del item 13, pues puede afectar significativamente a los resultados.

Las correlaciones entre las subescalas resultaron significativas en todos los casos. Al igual que ocurre con el MBI-HSS, la correlación más intensa se establece entre agotamiento y cinismo. Este resultado, similar a los obtenidos en casi todos los estudios revisados, sugiere que Agotamiento y Cinismo, tal vez, puedan ser integrados en una sola dimensión (lo que se ha denominado el "núcleo del burnout" en el MBI-HSS), y apoyaría la perspectiva del modelo bifactorial del MBI (Agotamiento-Cinismo vs. Eficacia profesional), que en algunos estudios ha obtenido índices de ajuste similares a los obtenidos por el modelo trifactorial.

Como conclusión podemos afirmar que, si bien esta adaptación del MBI-GS presenta debilidades (la dimensión de Cinismo se desdobló en dos factores y el item 13 puede ser ambivalente y poco consistente), los resultados obtenidos apoyan la estructura trifactorial de la escala. Alguna de estas debilidades son propias del instrumento, no específicas de la adaptación. Por tanto, la adaptación al castellano del MBI-GS reúne los suficientes requisitos de validez factorial y consistencia interna como para ser empleada en la estimación del síndrome de quemarse por el trabajo. No obstante, hay que tener presente que los resultados pueden estar influidos por las particularidades de la muestra utilizada para la validación (v.g., está compuesta en su mayor parte por hombres), por ello deben ser replicados en futuros estudios mediante análisis factorial confirmatorio. Aún así estos resultados tienen una gran proyección aplicada, pues nos facilitan un instrumento que permitirá evaluar el síndrome de quemarse por el trabajo en organizaciones que no son del sector servicios y en profesionales que no trabajan hacia perso- 
nas. Esta evaluación ayudará a prevenir algunos de los principales riesgos psicosociales del trabajo $\mathrm{y}$, consecuentemente, permitirá disminuir el absentismo en el trabajo y mejorará la calidad de vida laboral de los trabajadores.

\section{Referencias}

1. Maslach C, Jackson SE, Leiter MP. Maslach Burnout Inventory Manual. 30 edición. Palo Alto, C alifornia: Consulting Psychologists Press, 1996. 2. Schwab RL. Burnout in education. En: Maslach C, Jackson SE, comp. Maslach Burnout Inventory Manual. 20 edición. Palo Alto, California: Consulting Psychologists Press, 1986:18-22.

3. Maslach C, Jackson SE. Maslach Burnout Inventory Manual. 10 edición. Palo Alto, California: Consulting Psychologists Press, 1981.

4. Maslach C, Schaufeli W B. Historical and conceptual development of burnout. En: Schaufeli W B, Maslach C, Marek T, ed. Professional burnout: Recent developments in theory and research. Londres:Taylor \& Francis, 1993:1-16

5. Schaufeli W B, Leiter MP, Maslach, C, Jackson SE. The Maslach Burnout Inventory: General Survey (MBI-GS). En: Maslach C, Jackson SE, Leiter MP, ed. Maslach Burnout Inventory Manual. 30 edición. Palo Alto, California: Consulting Psychologists Press, 1996:19-26.
6. Leiter MP, Schaufeli W B. C onsistency of the burnout construct across occupations. Anxiety Stress Coping 1996;9:229-243.

7. Maslach C, Jackson SE. Maslach Burnout Inventory-Human Services Survey (MBI-HSS). En: Maslach C, Jackson SE, Leiter MP, ed. Maslach Burnout Inventory Manual. 30 edición. Palo Alto, California: Consulting Psychologists Press, 1996.

8. Schaufeli W B, Leiter MP, Kalimo R. The General Burnout Inventory: A self-reported questionnaire to assess burnout at the workplace. En: Leiter MP, ed. Extending the burnout construct: Reflecting changing career paths. Symposium APA/N IO SH Conference, W ork, Stress, \& Health=95: C reating a Healthier W orkplace; 1995 sept;W ashington, D.C.

9. Taris TW, Schreurs PJ, Schaufeli W B. Construct validity of the Maslach Burnout Inventory-General Survey:A two-sample examination of its factor structure and correlates. Work Stress 1999;13(3):223-237.

10. Schutte N ,Toppinen S, Kalimo R, Schaufeli W B.The factorial validity of the Maslach Burnout Inventory-General Survey (MBI-GS) across occupational groups and nations. J 0 ccup 0 rgan Psychol 2000;73:53-66.

11. Kaiser HF.The application of electronic computers to analysis factorial. Educ Psychol Mea 1960;20:141-151.

12.Tabachnick BG , Fidell LS. U sing multivariate statistics. 20 edición. N uevaYork: Harper Collins, 1989.

13. Comrey AL.A first course in factor analysis. Nueva York: Academic Press, 1973

14. N unnaly N C. Psychometric theory. N ueva York: MCG raw-Hill, 1978. 15. Gil-Monte PR, Peiró JM. Un estudio comparativo sobre criterios normativos y diferenciales para el diagnóstico del síndrome de quemarse por el trabajo ("burnout") en España según el MBI-HSS. Rev PsicologiaTrabajo O rgan 2000; 16(2):135-149. 